\title{
Hypersurfaces with degenerate duals and the Geometric Complexity Theory Program
}

Joseph M. Landsberg, Laurent Manivel and Nicolas Ressayre*

\begin{abstract}
We determine set-theoretic defining equations for the variety $\operatorname{Dual}_{k, d, N} \subset \mathbb{P}\left(S^{d} \mathbb{C}^{N}\right)$ of hypersurfaces of degree $d$ in $\mathbb{C}^{N}$ that have dual variety of dimension at most $k$. We apply these equations to the Mulmuley-Sohoni variety $\overline{\mathrm{GL}_{n^{2}} \cdot\left[\operatorname{det}_{n}\right]} \subset \mathbb{P}\left(S^{n} \mathbb{C}^{n^{2}}\right)$, showing it is an irreducible component of the variety of hypersurfaces of degree $n$ in $\mathbb{C}^{n^{2}}$ with dual of dimension at most $2 n-2$. We establish additional geometric properties of the Mulmuley-Sohoni variety and prove a quadratic lower bound for the determinantal border-complexity of the permanent.
\end{abstract}

Mathematics Subject Classification (2010). 68Q17, 14N05, 20G05, 14L30.

Keywords. Dual variety, geometric complexity theory, determinant, permanent.

\section{Introduction}

1.1. The GCT program. A classical problem in linear algebra is to determine or bound the smallest integer $n$ such that the permanent of an $m \times m$ matrix may be realized as an affine linear projection of the determinant of an $n \times n$ matrix. L. Valiant [7] proposed using this problem as an algebraic analog of the problem of comparing the complexity classes $\boldsymbol{P}$ and $\boldsymbol{N P}$. Denote this value of $n$ by $\operatorname{dc}\left(\operatorname{perm}_{m}\right)$. He conjectured that $\mathrm{dc}\left(\operatorname{perm}_{m}\right)$ grows faster than any polynomial in $m$. The best known lower bound is $\operatorname{dc}\left(\operatorname{perm}_{m}\right) \geq \frac{m^{2}}{2}$, which was proved in [3].

The definition of $\mathrm{dc}\left(\right.$ perm $\left._{m}\right)$ may be rephrased as follows: let $\ell$ be a linear coordinate on $\mathbb{C}$, let $\mathbb{C} \oplus M_{m}(\mathbb{C})^{*} \subset M_{n}(\mathbb{C})^{*}$ be any linear inclusion, where $M_{n}(\mathbb{C})$ denotes the space of complex $n \times n$ matrices; then $\operatorname{dc}\left(\right.$ perm $\left._{m}\right)$ is the smallest $n$ such that $\ell^{n-m} \operatorname{perm}_{m} \in \operatorname{End}\left(M_{n}(\mathbb{C})\right) \cdot \operatorname{det}_{n}$. Here $u \in \operatorname{End}\left(M_{n}(\mathbb{C})\right)$ acts by $\left(u \cdot \operatorname{det}_{n}\right)(M):=\operatorname{det}_{n}(u(M))$.

K. Mulmuley and M. Sohoni (see [4], [5]) have proposed to study the function $\overline{\mathrm{dc}}\left(\right.$ perm $\left._{m}\right)$, which is the smallest $n$ such that $\left[\ell^{n-m}\right.$ perm $\left._{m}\right]$ is contained in the orbit closure $\overline{\mathrm{GL}_{n^{2}} \cdot\left[\operatorname{det}_{n}\right]} \subset \mathbb{P}\left(S^{n}\left(M_{n}(\mathbb{C})\right)^{*}\right)$. Here $S^{n}\left(M_{n}(\mathbb{C})\right)^{*}$ denotes the homo-

\footnotetext{
* Landsberg supported by NSF grant DMS-0805782. NR supported by the French National Research Agency (ANR-09-JCJC-0102-01).
} 
geneous polynomials of degree $n$ on $M_{n}(\mathbb{C})$. The best known lower bound on this function had been linear. Note that $\overline{\mathrm{dc}}\left(\operatorname{perm}_{m}\right) \leq \mathrm{dc}\left(\operatorname{perm}_{m}\right)$, the potential difference being the added flexibility of limiting polynomials in $\overline{\mathrm{GL}_{n^{2}} \cdot\left[\operatorname{det}_{n}\right]}$ that are not in $\operatorname{End}\left(M_{n}(\mathbb{C})\right) \cdot\left[\operatorname{det}_{n}\right]$. Our main result about $\overline{\mathrm{dc}}\left(\operatorname{perm}_{m}\right)$ is the following quadratic bound.

Theorem 1.1.1. $\overline{\mathrm{dc}}\left(\operatorname{perm}_{m}\right) \geq \frac{m^{2}}{2}$.

Consider the ideal of regular functions on $S^{n}\left(M_{n}(\mathbb{C})^{*}\right)$ that are zero on $\overline{\mathrm{GL}_{n^{2}} \cdot\left[\operatorname{det}_{n}\right]}$. We construct an explicit sub-GL $n^{2}$-module $V_{n}$ in this ideal which has the following properties.

Theorem 1.1.2. (1) The $\mathrm{GL}_{n^{2}}$-module $V_{n}$ contains an irreducible module of highest weight

$$
2 n(n-1)(n-2) \omega_{1}+\left(2 n^{2}-4 n-1\right) \omega_{2}+2 \omega_{2 n+1}
$$

and $V_{n}$ is a subspace of the space of homogeneous polynomials of degree $n(n-1)$ on $S^{n}\left(M_{n}(\mathbb{C})\right)^{*}$.

(2) The variety $\overline{\mathrm{GL}_{n^{2}} \cdot\left[\operatorname{det}_{n}\right]}$ is an irreducible component of the zero locus $\mathscr{D}_{n}$ of $V_{n}$.

Theorem 1.1.2 provides the first explicit module of equations in the ideal of $\overline{\mathrm{GL}_{n^{2}} \cdot\left[\operatorname{det}_{n}\right]}$. However, the dimension $\operatorname{dim}\left(\mathscr{D}_{n}\right)$ grows exponentially with $n$, whereas $\operatorname{dim}\left(\overline{\mathrm{GL}_{n^{2}} \cdot\left[\mathrm{det}_{n}\right]}\right)$ is on the order of $n^{4}$. In particular, $\mathscr{D}_{n}$ has other irreducible components, one of which is described in $\S 4$. A more precise statement than Theorem 1.1.2 is Theorem 3.1.1, which implies that our equations provide a full set of local equations of $\overline{\mathrm{GL}_{n^{2}} \cdot\left[\operatorname{det}_{n}\right]}$ around $\left[\operatorname{det}_{n}\right]$.

1.2. Dual varieties. One can similarly define $\operatorname{dc}(P), \overline{\operatorname{dc}}(P)$ for an arbitrary polynomial $P$ of degree $n$ in $N$ variables. Such a polynomial, if nonzero, defines a hypersurface $Z(P) \subset \mathbb{P}^{N-1}$. If $P$ is reduced, the Zariski closure of the set of tangent hyperplanes to this hypersurface is a subvariety $Z(P)^{*}$ of the dual projective space, called the dual variety of $Z(P)$. For general such $P, Z(P)^{*}$ is a hypersurface.

Theorem 1.2.1. For any irreducible polynomial $P$,

$$
\overline{\operatorname{dc}}(P) \geq \frac{\operatorname{dim} Z(P)^{*}+1}{2} .
$$

Theorem 1.2.1 is obtained by partially solving a question in classical algebraic geometry (Theorem 2.3.1): find set-theoretic defining equations for the variety Dual $_{k, d, N} \subset \mathbb{P}\left(S^{d} \mathbb{C}^{N}\right)$ of hypersurfaces of degree $d$ in $\mathbb{C} \mathbb{P}^{N-1}$ whose dual variety has dimension at most $k$. Usually one only discusses dual varieties of irreducible varieties, in fact there are several possible definitions of the dual of a reducible variety. 
Thus it would be more precise to say our equations describe Dual $_{k, d, N}$ locally on the set of irreducible hypersurfaces.

While it was generally understood that $\operatorname{End}\left(M_{n}(\mathbb{C})\right) \cdot\left[\operatorname{det}_{n}\right] \subset \overline{\mathrm{GL}_{n^{2}} \cdot\left[\operatorname{det}_{n}\right]}$ was a proper inclusion, it had not been known if the difference was potentially significant. Proposition 3.5.1 exhibits an explicit codimension one $\mathrm{GL}_{n^{2}}(\mathbb{C})$-orbit that is contained in the boundary of $\overline{\mathrm{GL}_{n^{2}} \cdot\left[\operatorname{det}_{n}\right]}$ but not contained in $\operatorname{End}\left(\mathbb{C}^{n^{2}}\right) \cdot \operatorname{det}_{n}$, at least when $n$ is odd. In particular, we exhibit an explicit sequence of polynomials $P_{m}$ with $\overline{\mathrm{dc}}\left(P_{m}\right)<\operatorname{dc}\left(P_{m}\right)$.

\section{Hypersurfaces with degenerate dual varieties}

2.1. B. Segre's dimension formula. Let $W$ be a complex vector space of dimension $N$, and $P \in S^{d} W^{*}$ a homogeneous polynomial of degree $d$. Let $Z(P) \subset \mathbb{P} W$ denote the hypersurface defined by $P$. If $P$ is irreducible, then $Z(P)$ and its dual variety $Z(P)^{*}$, the Zariski closure of the set of tangent hyperplanes to $Z(P)$, are both irreducible. The Segre dimension formula [2] states that

$$
\operatorname{dim} Z(P)^{*}=\operatorname{rank}\left(H_{P, w}\right)-2,
$$

where $H_{P, w}$ denotes the Hessian of $P$ at $w$, a general point of the affine cone over $Z(P)$. Recall that the Hessian can be defined, once a coordinate system on $W$ has been chosen, as the symmetric matrix of second partial derivatives of $P$. Intrinsically, it is just the quadratic form constructed from $P$ by polarization:

$$
H_{P, w}(X):=P(w, \ldots, w, X, X) \text {. }
$$

Segre's formula implies that $Z(P)^{*}$ has dimension less or equal to $k$ if and only if, for any $w \in W$ such that $P(w)=0$, and any $(k+3)$-dimensional subspace $F$ of $W$,

$$
\operatorname{det}\left(\left.H_{P, w}\right|_{F}\right)=0 .
$$

Equivalently (assuming $P$ is irreducible), for any such subspace $F$, the polynomial $P$ must divide $\operatorname{det}\left(\left.H_{P}\right|_{F}\right)$, a polynomial of degree $(k+3)(d-2)$.

Note that for polynomials in $N^{\prime}<N$ variables, the maximum rank of the Hessian is $N^{\prime}$ so in particular the determinant of the Hessian will vanish on any $F$ of dimension $N^{\prime}+1$.

2.2. Pairs of polynomials such that one divides the other. Consider two homogeneous polynomials $P, Q$ on $W=\mathbb{C}^{N}$, of respective degrees $d$, $e$. We determine equations on their coefficients that are implied by the condition that $P$ divides $Q$.

There is an obvious solution in the slightly different situation where $P$ and $Q$ are (non-homogeneous) polynomials in a single variable: one simply performs the 
Euclidian division of $Q$ by $P$ and requires that the remainder $R$ be zero. The ideal defined by this condition is described in [6].

In our situation, we can first restrict $P$ and $Q$ to some plane $L$ in $W$, and choose coordinates $x, y$ on $L$. The restricted polynomials $P_{L}$ and $Q_{L}$ are then binary forms in these coordinates. Then set $y=1$ and perform a Euclidean division on the resulting polynomials in $x$. After rehomogenization, we obtain

$$
Q_{L}(x, y)=P_{L}(x, y) D_{L}(x, y)+y^{e-d+1} R_{L}(x, y),
$$

where $R_{L}(x, y)$ is homogeneous of degree $d-1$. The condition $R_{L}=0$ depends on the choice of the coordinates $x$ and $y$, but up to scale, the coefficient $R_{L, d-1}$ of $x^{d-1}$ only depends on the choice of the coordinate $y$. That is, the condition $R_{L, d-1}=0$, considered as a polynomial equation in the coefficients of $P$ and $Q$, only depends on the choice of $L$ and of the line $D$ in $L$ defined by the equation $y=0$.

To make the connection with [6], write

$$
\begin{aligned}
Q_{L}(x, y) & =\sum_{i=0}^{e} q_{i} x^{i} y^{e-i}=q_{e} \prod_{k=1}^{e}\left(x-y \alpha_{k}\right), \\
P_{L}(x, y) & =\sum_{j=0}^{d} p_{j} x^{j} y^{d-j}=p_{d} \prod_{l=1}^{d}\left(x-y \beta_{l}\right) .
\end{aligned}
$$

Divide equation (1) by $P_{L}(x, y)$ and set $x=1$. We get an identity between power series in $y$, to which $D_{L}$ contributes only up to degree $e-d$. We conclude that $R_{L, d-1} / p_{d}$ is equal to the coefficient of $y^{e-d+1}$ in

$$
\frac{Q_{L}(1, y)}{P_{L}(1, y)}=\frac{q_{e} \prod_{k=1}^{e}\left(1-y \alpha_{k}\right)}{p_{d} \prod_{l=1}^{d}\left(1-y \beta_{l}\right)}=\frac{q_{e}}{p_{d}} \sum_{m \geq 0} s_{m}(\beta-\alpha) y^{m},
$$

where the last equality can be taken as a definition of the symmetric functions $s_{m}(\beta-\alpha)$. The condition that $R_{L, d-1}=0$ is thus equivalent to the condition that

$$
s_{e-d+1}(\beta-\alpha)=0 .
$$

In order to get a polynomial equation in the coefficients of $Q_{L}$ and $P_{L}$, we modify the expression slightly. Write

$$
\frac{Q_{L}(1, y)}{P_{L}(1, y)}=\frac{Q_{L}(1, y)}{p_{d}(1+\pi(y))}=\frac{Q_{L}(1, y)}{p_{d}} \sum_{m \geq 0}(-1)^{m} \pi(y)^{m},
$$

where $\pi(y)=\sum_{j=1}^{d} \frac{p_{d-j}}{p_{d}} y^{j}$. Therefore, the coefficient of $y^{e-d+1}$ can be expressed as

$$
\widehat{R}(Q, P):=\frac{1}{p_{d}} \sum_{i=0}^{e} q_{i} \sum_{j_{1}+\cdots+j_{r}=-d+1+i}(-1)^{r} \frac{p_{d-j_{1}} \cdots p_{d-j_{r}}}{p_{d}^{r}} .
$$


In that sum the maximal value of $r$ is $e-d+1$, so we make it a polynomial by multiplying by $p_{d}^{e-d+2}$. We conclude that $R_{L, d-1}=0$ is equivalent to the condition that

$$
\sum_{j_{1}+\cdots+j_{r}=-d+1+i}(-1)^{r} q_{i} p_{d-j_{1}} \cdots p_{d-j_{r}} p_{d}^{e-d+1-r}=0 .
$$

This condition is linear in the coefficients of $Q_{L}$, and of degree $e-d+1$ in those of $P_{L}$. It depends, as we have seen, on the choice of a preferred coordinate on $L$, in particular, on the choice of the line $D$ defined by this coordinate.

Note the following behavior under rescaling:

$$
\widehat{R}(\alpha Q(x, \lambda y), \beta P(x, \lambda y))=\alpha \beta^{e-d+1} \lambda^{e-d+1} \hat{R}(Q, P) .
$$

2.3. Equations for hypersurfaces with degenerate duals. We apply the results of the preceding section to the case where $Q=\operatorname{det}\left(\left.H_{P}\right|_{F}\right)$, whose degree equals $e=(k+3)(d-2)$. Recall that $F \subset W$ is a subspace of dimension $k+3$. Once $F$ has been chosen, we obtain a family of equations depending, up to scale, only on the choice of a plane $L$ in $W$ and a line $D$ in $L$. In particular, if $F$ contains $L$ we get an equation depending only on the partial flag $D \subset L \subset F$. This equation must therefore be a highest weight vector in some module of polynomials on $S^{n} W^{*}$, and its highest weight must be of the form $a \omega_{1}+b \omega_{2}+c \omega_{k+3}$.

Consider a basis adapted to $D \subset L \subset F$ and let $(x, y, z, w)=\left(x, y, z^{i}, w^{s}\right)$ denote its dual basis. Consider a diagonal matrix $T:=\left(t_{x}, t_{y}, t_{z} \operatorname{Id}_{F / L}, t_{w} \operatorname{Id}_{W / F}\right)$. Under rescaling

$$
(T . P)(x, y, 0,0)=t_{x}^{-n} P\left(x, t_{x} t_{y}^{-1} y, 0,0\right) .
$$

Moreover, the matrix of $\left.H_{T . P}\right|_{F}$ is obtained from that of $\left.H_{P}\right|_{F}$ by substituting $(x, y, z, w)$ by $\left(t_{x}^{-1} x, t_{y}^{-1} y, t_{z}^{-1} z, t_{w}^{-1} w\right)$ and multiplying the first row and column by $t_{x}^{-1}$, the second row and column by $t_{y}^{-1}$ and the other rows and columns by $t_{z}^{-1}$. It follows that $\operatorname{det}\left(\left.H_{T . P}\right|_{F}\right)$ is obtained from $\operatorname{det}\left(\left.H_{P}\right|_{F}\right)$ by substituting $\left(t_{x}^{-1} x, t_{y}^{-1} y, t_{z}^{-1} z, t_{w}^{-1} w\right)$ in for $(x, y, z, w)$ and by multiplying the result by

$$
t_{x}^{-2} t_{y}^{-2} t_{z}^{-2(k+1)} \text {. }
$$

In summary,

$$
\operatorname{det}\left(\left.H_{T . P}\right|_{F}\right)(x, y, 0,0)=t_{x}^{-2} t_{y}^{-2} t_{z}^{-2(k+1)} t_{x}^{-e} \operatorname{det}\left(\left.H_{P}\right|_{F}\right)\left(x, t_{x} t_{y}^{-1} y, 0,0\right) .
$$

From equations (3), (4) and (5), the vector of exponents of the action of $T$ on our equation is:

$$
\left(\begin{array}{c}
2+e+(d-1)(e-d+1) \\
e-d+3 \\
2(k+1)
\end{array}\right)
$$


This vector should be

$$
\left(\begin{array}{c}
a+b+c \\
b+c \\
c(k+1)
\end{array}\right)
$$

We deduce

$$
\begin{aligned}
& a=-e+3 d-2+d e-d^{2}=(d-1)(d-2)(k+2), \\
& b=e-d+1=d(k+2)-2 k-5 \\
& c=2 .
\end{aligned}
$$

Note that $a+2 b+(k+3) c=d(d-1)(k+2)$ so this module occurs in $W^{\otimes d(d-1)(k+2)}$.

Define Dual $_{k, d, N} \subset \mathbb{P}\left(S^{d} W^{*}\right)$ as the Zariski closure of the set of irreducible hypersurfaces of degree $d$ in $\mathbb{P} W \simeq \mathbb{P}^{N-1}$, whose dual variety has dimension at most $k$.

Theorem 2.3.1. The variety Dual $k, d, N \subset \mathbb{P}\left(S^{d}\left(\mathbb{C}^{N}\right)^{*}\right)$ has equations given by a copy of the $\mathrm{SL}_{N}$-module with highest weight

$$
\Omega(k, d)=(d-1)(d-2)(k+2) \omega_{1}+(d(k+2)-2 k-5) \omega_{2}+2 \omega_{k+3} .
$$

These equations have degree $(k+2)(d-1)$.

Note that when we constructed our equations, we did not suppose that $L$ was contained in $F$. This indicates that the module generated by these equations should in fact be larger than the single module with highest weight $\Omega(k, d)$.

Set theoretically, these equations suffice to define Dual $_{k, d, N}$ locally, at least on the open subset parametrizing irreducible hypersurfaces $Z(P) \subset \mathbb{P}(W)$. Indeed, once the plane $L$ is fixed, by varying the line $D$ one obtains a family of equations expressing the condition that $P_{L}$ divides $Q_{L}$, respectively the restrictions to $L$ of the polynomials $P$ and $Q=\operatorname{det}\left(\left.H_{P}\right|_{F}\right)$. But $P$ divides $Q$ if and only if restricted to each plane $P$ divides $Q$, so our conditions imply that the dual variety of the irreducible hypersurface $Z(P)$ has dimension at most $k$. On the other hand, if $P$ is not reduced, then these equations can vanish even if the dual of $P_{\text {red }}$ is non-degenerate. For example, if $P=R^{2}$ where $R$ is a quadratic polynomial of $\operatorname{rank} 2 s$, then $\operatorname{det}\left(H_{P}\right)$ is a multiple of $R^{2 s}$.

\subsection{Polynomials of the form $\ell^{d-m} R$}

Lemma 2.4.1. Let $U=\mathbb{C}^{M}$ and $L=\mathbb{C}$, let $R \in S^{m} U^{*}$ be irreducible, let $\ell \in L^{*}$ be nonzero, let $U^{*} \oplus L^{*} \subset W^{*}$ be a linear inclusion, and let $P=\ell^{d-m} R \in S^{d} W^{*}$. If $[R] \in \operatorname{Dual}_{\kappa, m, M}$ and $[R] \notin \operatorname{Dual}_{\kappa-1, m, M}$, then $[P] \in \operatorname{Dual}_{\kappa, d, N}$ and $[P] \notin$ Dual $_{\kappa-1, d, N}$. 
Proof. Choose a basis $u_{1}, \ldots, u_{M}, v, w_{M+2}, \ldots, w_{N}$ of $W$ so that

$$
\left(U^{*}\right)^{\perp}=\left\langle w_{M+2}, \ldots, w_{N}\right\rangle
$$

and

$$
\left(L^{*}\right)^{\perp}=\left\langle u_{1}, \ldots, u_{M}, w_{M+2}, \ldots, w_{N}\right\rangle
$$

Let $c=(d-m)(d-m-1)$. In these coordinates, we have the matrix in the following $(M, 1, N-M-1) \times(M, 1, N-M-1)$-block form:

$$
H_{P}=\left(\begin{array}{ccc}
\ell^{d-m} H_{R} & 0 & 0 \\
0 & c \ell^{d-m-2} R & 0 \\
0 & 0 & 0
\end{array}\right) .
$$

First note that $\operatorname{det}_{M+1}\left(\left.H_{P}\right|_{F}\right)$ for any $F=\mathbb{C}^{M+1}$ is either zero or a multiple of $P$. If $\operatorname{dim} Z(R)^{*}=M-2$ (the expected dimension), then for a general $F=\mathbb{C}^{M+1}$, $\operatorname{det}_{M}\left(\left.H_{P}\right|_{F}\right)$ will not be a multiple of $P$, and more generally if $\operatorname{dim} Z(R)^{*}=\kappa$, then for a general $F=\mathbb{C}^{\kappa+2}, \operatorname{det}_{\kappa+2}\left(\left.H_{P}\right|_{F}\right)$ will not be a multiple of $P$ but for any $F=\mathbb{C}^{\kappa+3}, \operatorname{det}_{\kappa+3}\left(\left.H_{P}\right|_{F}\right)$ will be a multiple of $P$.

\section{The orbit of the determinant}

3.1. Statement of the main result. Let $W=M_{n}(\mathbb{C})$, the space of complex matrices of size $n$. Its dimension is $N=n^{2}$. The hypersurface in $\mathbb{P} W$ defined by the determinant is dual to the variety of rank one matrices, the Segre product $\mathbb{P}^{n-1} \times$ $\mathbb{P}^{n-1} \subset \mathbb{P}^{N-1}$.

Intuitively, a deformation of the determinant hypersurface, subject to the condition that its dual remains of dimension $2 n-2$, should have a deformation of the Segre as its dual variety. But the Segre is rigid, its only deformations in $\mathbb{P} W^{*}$ are translates by projective automorphisms. Hence the only deformations of the determinant hypersurface, with duals of the same dimension, should be translates by projective automorphisms as well.

The problem with this intuitive argument is that the dual map can be highly discontinuous, especially in the presence of singularities, and the determinant hypersurface is very singular. Nevertheless, the conclusion turns out to be correct:

Every small deformation of the determinant hypersurface, with dual variety of the same dimension, is a translate by a projective automorphism.

We will prove a more precise statement. For a polynomial $P$ of degree $n$ on $W$, and a $k$-dimensional subspace $F$ of $W$, we have expressed the condition that $P$ divides $\operatorname{det}\left(\left.H_{P}\right|_{F}\right)$ in terms of polynomial equations of degree $(k+2)(n-1)$. These equations define a subscheme $\operatorname{Dual}_{k, n, N} \subset \mathbb{P} S^{n} W^{*}$, supported on the variety Dual $_{k, n, N}$ and possibly other reducible hypersurfaces. 
Theorem 3.1.1. The scheme Dual $2 n-2, n, n^{2}$ is smooth at $\left[\operatorname{det}_{n}\right]$, and the $\mathrm{PGL}_{n^{2}}$-orbit closure of $\left[\operatorname{det}_{n}\right]$ is an irreducible component of Dual $_{2 n-2, n, n^{2}}$.

In particular, Theorem 3.1.1 implies that the $\mathrm{SL}(W)$-module of highest weight $\Omega(2 n-2, n)$ given by Theorem 2.3.1 gives local equations at [det $n$ of $\overline{\mathrm{GL}_{n^{2}} \cdot\left[\operatorname{det}_{n}\right]}$, of degree $2 n(n-1)$. Since $\operatorname{Dual}_{k, n, N}$ always contains the variety of degree $n$ hypersurfaces which are cones over a linear space of dimension $N-k-1$, the zero set of the equations is strictly larger than $\overline{\mathrm{GL}_{n^{2}} \cdot\left[\operatorname{det}_{n}\right]}$. The so-called subspace variety of cones has dimension $\left(\begin{array}{c}k+n+1 \\ n\end{array}\right)+(k+2)(N-k-2)-1$. For $k=2 n-2, N=n^{2}$, this dimension is bigger than the dimension of the orbit of [det $\left.{ }_{n}\right]$, and therefore Dual $_{2 n-2, n, n^{2}}$ is not irreducible. We have not yet been able to find equations that separate the orbit of $\left[\operatorname{det}_{n}\right]$ from the other components of $\mathrm{Dual}_{2 n-2, n, n^{2}}$.

3.2. Consequences regarding Kronecker coefficients. A copy of the module with highest weight $2 n(n-1)(n-2) \omega_{1}+\left(2 n^{2}-4 n-1\right) \omega_{2}+2 \omega_{2 n+1}$ in $S^{2 n(n-1)}\left(S^{n} \mathbb{C}^{n^{2}}\right)$ is in the ideal of $\overline{\mathrm{GL}(W) \cdot\left[\operatorname{det}_{n}\right]}$.

The program suggested in [5] was to separate the determinant and permanent by finding $\mathrm{SL}(W)$-modules in the ideal of $\overline{\mathrm{GL}_{n^{2}} \cdot\left[\mathrm{det}_{n}\right]}$ such that their entire isotypic component was in the ideal. (Also see [1] for explicit statements regarding Kronecker coefficients needed to carry out the program.) This does not occur for the module with highest weight $2 n(n-1)(n-2) \omega_{1}+\left(2 n^{2}-4 n-1\right) \omega_{2}+2 \omega_{2 n+1}$.

For example, when $n=3$, the module with highest weight $12 \omega_{1}+5 \omega_{2}+2 \omega_{7}$ occurs with multiplicity six in $S^{12}\left(S^{3} \mathbb{C}^{9}\right)$, but only one copy of it is in the ideal.

3.3. Computing the Zariski tangent space. We differentiate the condition that $P$ divides $\operatorname{det}\left(\left.H_{P}\right|_{F}\right)$ for each $F$. That is, write $\operatorname{det}\left(\left.H_{P}\right|_{F}\right)=P Q_{F}$ for some polynomial $Q_{F}$, and consider a curve $P_{\epsilon}=P+\epsilon \pi+\epsilon^{2} \tau+O\left(\epsilon^{3}\right)$, inducing a curve $Q_{F, \epsilon}=Q_{F}+\epsilon Q_{F}^{\prime}+O\left(\epsilon^{2}\right)$. Up to $O\left(\epsilon^{2}\right), H_{P}$ becomes $H_{P}+\epsilon H_{\pi}$ and we deduce the identity

$$
\left.\operatorname{det}\left(H_{P}, \ldots, H_{P}, H_{\pi}\right)\right|_{F}=\pi Q_{F}+P Q_{F}^{\prime} .
$$

To exploit (6), let $[w]$ be a general point of the hypersurface $Z(P)$, so the rank of the quadratic form $H_{P, w}$ is exactly $k+2$. Let $X$ belong to the kernel of $H_{P, w}$. Let $F^{\prime}$ be a $(k+2)$-dimensional subspace of $W$, transverse to the kernel of $H_{P, w}$, and let $F=F^{\prime} \oplus \mathbb{C} X$. Now compute $\left.\operatorname{det}\left(H_{P}, \ldots, H_{P}, H_{\pi}\right)\right|_{F}$ at $w$. In terms of bases adapted to the flag $F^{\prime} \subset F \subset W$, the matrix of $H_{P, w}$ has zeros in its last row and column, since they correspond to $X$, which belongs to the kernel. Removing this row and column yields an invertible matrix, corresponding to $\left.H_{P, w}\right|_{F^{\prime}}$, as $F^{\prime}$ is transverse to the kernel.

Now, $\left.\operatorname{det}\left(H_{P}, \ldots, H_{P}, H_{\pi}\right)\right|_{F}$ evaluated at $w$ is the sum of the $k+3$ determinants obtained by considering the matrix of $\left.H_{P, w}\right|_{F}$ and replacing one column by the 
corresponding column of $\left.H_{\pi, w}\right|_{F}$. If this column is not the last one, this determinant remains with a zero column, hence equals zero. In case the replaced column is the last one, since the last row of the matrix of $\left.H_{P, w}\right|_{F}$ vanishes, the resulting determinant is equal to the determinant of the upper-left block, $\operatorname{det}\left(\left.H_{P, w}\right|_{F^{\prime}}\right)$, multiplied by the lower-right entry of $\left.H_{\pi, w}\right|_{F}$, that is, $H_{\pi, w}(X)=\pi(w, \ldots, w, X, X)$. Equation (6) becomes

$$
\operatorname{det}\left(\left.H_{P, w}\right|_{F^{\prime}}\right) H_{\pi, w}(X)=\pi(w) Q_{F}(w) .
$$

Note that $Q_{F}(w)$ depends on both $w$ and $X$ (since $F$ depends on $\left.X\right)$, $\operatorname{but} \operatorname{det}\left(\left.H_{P, w}\right|_{F^{\prime}}\right)$ only depends on $w$.

Now specialize (7) to the case $P=\operatorname{det}_{n}$. Then $w$ must be a matrix of rank exactly $n-1$. Write $W=E \otimes E^{*}$, and as such, it is naturally self-dual via the involution $e \otimes \phi \mapsto \phi \otimes e$. For $w \in W$, write $w^{*} \in E^{*} \otimes E=W^{*}$ for the image of $w$ under the involution.

Lemma 3.3.1. Let $w$ be a matrix of rank exactly $n-1$. Then the singular locus of the quadratic form $H_{\operatorname{det}_{n}, w},\left(H_{\operatorname{det}_{n}, w}\right)_{\operatorname{sing}}$ is the space of $n \times n$ matrices $X$ such that:

$$
\text { 1) } X(E) \subset w(E), \text { 2) } X\left(E^{*}\right) \subset w\left(E^{*}\right), \text { 3) } w^{*}(X)=0 \text {. }
$$

Proof. Write $w=\phi_{1} \otimes e_{1}+\cdots+\phi_{n-1} \otimes e_{n-1}$, for some collection $e_{1}, \ldots, e_{n-1}$ of independent vectors in $E=\mathbb{C}^{n}$, and some collection $\phi_{1}, \ldots, \phi_{n-1}$ of independent linear forms. We complete these collections into bases by adding a vector $e_{n}$ and a linear form $\phi_{n}$. Consider an endomorphism $X=\sum_{1 \leq i, j \leq n} x_{i j} \phi_{i} \otimes e_{j}$. An easy computation yields

$$
H_{\operatorname{det}_{n}, w}(X)=\operatorname{det}(w, \ldots, w, X, X)=\sum_{i=1}^{n-1}\left(x_{n n} x_{i i}-x_{n i} x_{i n}\right)
$$

Taking the exterior derivative shows that the singular locus of the quadratic form $H_{\operatorname{det}_{n}, w}$ is defined by the conditions $x_{n i}=x_{i n}=0$ for $1 \leq i \leq n$, and $\sum_{i=1}^{n-1} x_{i i}=0$. The first identities are equivalent to the conditions $\operatorname{Im}(X) \subset \operatorname{Im}(w)$ and $\operatorname{Ker}(X) \supset$ $\operatorname{Ker}(w)$. The third one is the condition $w^{*}(X)=0$.

We summarize our analysis:

Lemma 3.3.2. Suppose that $\pi \in S^{n} W^{*}$ belongs to the affine Zariski tangent space $\widehat{T}_{\left[\operatorname{det}_{n}\right]} \mathcal{D u a l}_{2 n-2, n, n^{2}}$. Then for any matrix $w$ of rank $n-1$, and any $X \in\left(H_{\operatorname{det}_{n}, w}\right)_{\operatorname{sing}}$,

$$
H_{\pi, w}(X)=c_{X, w} \pi(w),
$$

for some scalar $c_{X, w}$ that does not depend on $\pi$. 
3.4. Immanants. Recall that each partition $\lambda$ of $n$ defines an irreducible representation $[\lambda]$ of the symmetric group $\widetilde{S}_{n}$, hence a character $\chi_{\lambda}$. The immanant $\operatorname{IM}_{\lambda}$ is the degree $n$ polynomial on $M_{n}$ defined by the formula

$$
\operatorname{IM}_{\lambda}(X)=\sum_{\sigma \in \mathbb{S}_{n}} \chi_{\lambda}(\sigma) x_{1 \sigma(1)} \cdots x_{n \sigma(n)} .
$$

For example, $[n]$ is the trivial representation and $\mathrm{IM}_{(n)}$ is the permanent; $\left[1^{n}\right]$ is the sign representation and $\mathrm{IM}_{\left(1^{n}\right)}$ is the determinant.

Write $M_{n}(\mathbb{C})=A^{*} \otimes B$ for two copies $A, B$ of $\mathbb{C}^{n}$. Since $\left[\operatorname{det}_{n}\right]$ is preserved by the action of $\mathrm{GL}(A) \times \mathrm{GL}(B)$ by left-right multiplication, this is also the case of the Zariski tangent space at $\left[\operatorname{det}_{n}\right]$ of the $\mathrm{GL}_{n^{2}}$-invariant scheme $\operatorname{Dual}_{2 n-2, n, n^{2}}$. But as a $\mathrm{GL}(A) \times \mathrm{GL}(B)$-module,

$$
S^{n}\left(A^{*} \otimes B\right)^{*}=\bigoplus_{\lambda} S_{\lambda} A \otimes S_{\lambda} B^{*},
$$

where the sum is over all partitions of $n$. Since this decomposition is multiplicity free, the submodule $\widehat{T}_{\left[\operatorname{det}_{n}\right]} \mathscr{D} u a l_{2 n-2, n, n^{2}}$ must be the direct sum of some of the components:

$$
\widehat{T}_{\left[\operatorname{det}_{n}\right]} \mathscr{D u a l}_{2 n-2, n, n^{2}}=\bigoplus_{\lambda \in P_{n}} S_{\lambda} A \otimes S_{\lambda} B^{*},
$$

for some set of partitions $P_{n}$ to be determined. Note that $\mathrm{IM}_{\lambda}$ is contained in the component $S_{\lambda} A \otimes S_{\lambda} B^{*}$. Therefore $\lambda$ belongs to $P_{n}$ if and only if $\mathrm{IM}_{\lambda}$ belongs to $\widehat{T}_{\left[\operatorname{det}_{n}\right]}$ Dual $_{2 n-2, n, n^{2}}$.

We apply Lemma 3.3.2 as follows. Start with a matrix $w$ of rank $n-1$, which we write as $\sum_{i=1}^{n} e_{i}^{*} \otimes c_{i}$. There is a dependence relation between $c_{1}, \ldots, c_{n}$, which we can suppose to be of the form $c_{n}=\sum_{i=1}^{n-1} \mu_{i} c_{i}$. Then $w=\sum_{i=1}^{n-1}\left(e_{i}^{*}+\mu_{i} e_{n}^{*}\right) \otimes c_{i}$. By Lemma 3.3.1, $\left(H_{\operatorname{det}_{n}, w}\right)_{\text {sing }}$ can then be described as the set of all

$$
X=\sum_{i=1}^{n-1}\left(e_{i}^{*}+\mu_{i} e_{n}^{*}\right) \otimes\left(\sum_{j=1}^{n-1} \zeta_{i}^{j} c_{j}\right),
$$

where $\sum_{i=1}^{n-1} \zeta_{i}^{i}=0$. In bases, the first $n-1$ columns $c_{1}^{\prime}, \ldots, c_{n-1}^{\prime}$ of $X$ are linear combinations of the columns of $w$, and $c_{n}^{\prime}=\sum_{i=1}^{n-1} \mu_{i} c_{i}^{\prime}$ is then given by the same linear combination as for the last column of $w$. We can thus write the entries of $X$ as

$$
x_{i}^{k}=\sum_{j=1}^{n-1} \zeta_{i}^{j} w_{j}^{k}, i<n, \quad x_{n}^{k}=\sum_{i=1}^{n-1} \mu_{i} x_{i}^{k} .
$$

Substituting these expressions into $H_{\mathrm{IM}_{\lambda}, w}(X)=\operatorname{IM}_{\lambda}(w, \ldots, w, X, X)$ yields a polynomial $\operatorname{IM}_{\lambda}\left(\zeta, w^{\prime}, \mu\right)$ which is quadratic in the $\zeta_{i}^{j}$ and of degree $n$ in the coeffi- 
cients $w_{j}^{k}, j<n$, of the first $n-1$ columns of $w$, denoted by $w^{\prime}$. Explicitly,

$$
\begin{aligned}
H_{\mathrm{IM}_{\lambda}, w} & (X) \\
= & \operatorname{IM}_{\lambda}\left(\zeta, w^{\prime}, \mu\right) \\
= & \sum_{i<j} \sum_{p, q} \zeta_{i}^{p} \zeta_{j}^{q}\left(\sum_{k, \sigma} \mu_{k} \chi_{\lambda}(\sigma) w_{1}^{\sigma(1)} \cdots w_{p}^{\sigma(i)} \cdots w_{q}^{\sigma(j)} \cdots w_{n-1}^{\sigma(n-1)} w_{k}^{\sigma(n)}\right) \\
& +\sum_{i, j} \sum_{p, q} \zeta_{i}^{p} \zeta_{j}^{q}\left(\sum_{\sigma} \mu_{j} \chi_{\lambda}(\sigma) w_{1}^{\sigma(1)} \cdots w_{p}^{\sigma(i)} \cdots w_{j}^{\sigma(j)} \cdots w_{n-1}^{\sigma(n-1)} w_{q}^{\sigma(n)}\right) .
\end{aligned}
$$

On the other hand, expressing the last column of $w$ in terms of the first ones, $\operatorname{IM}_{\lambda}(w)$ becomes a polynomial $\operatorname{IM}_{\lambda}\left(w^{\prime}, \mu\right)$, of degree $n$ in $w^{\prime}$ :

$$
\operatorname{IM}_{\lambda}(w)=\operatorname{IM}_{\lambda}\left(w^{\prime}, \mu\right)=\sum_{k, \sigma} \mu_{k} \chi_{\lambda}(\sigma) w_{1}^{\sigma(1)} \cdots w_{k}^{\sigma(k)} \cdots w_{n-1}^{\sigma(n-1)} w_{k}^{\sigma(n)} .
$$

By Lemma 3.3.2, for each choice of $\mu$, the vanishing of $\operatorname{IM}_{\lambda}\left(w^{\prime}, \mu\right)$ implies the vanishing of $\operatorname{IM}_{\lambda}\left(\zeta, w^{\prime}, \mu\right)$. Since they are both homogeneous of degree $m$ in $w^{\prime}$, they must be proportional.

This gives many relations, one for each quadratic monomial in the $\zeta$ 's (but recall the relation $\left.\sum_{i=1}^{n-1} \zeta_{i}^{i}=0\right)$. We will need only a small subset of them:

Lemma 3.4.1. Suppose that $\mathrm{IM}_{\lambda}$ belongs to $\widehat{T}_{\left[\operatorname{det}_{n}\right]} \mathcal{D u a l}_{2 n-2, n, n^{2}}$. Then for any permutation $\sigma$, and any triple of distinct integers $i, p, q$ smaller than $n$, one has the relations

$$
\sum_{\tau \in\langle(i p),(q n)\rangle} \chi_{\lambda}(\sigma \tau)=0
$$

Here $\langle(i p),(q n)\rangle$ denotes the group of permutations generated by the two simple transpositions $(i p)$ and $(q n)$. This group has order four, hence we get a collection of four term relations between the values of the character $\chi_{\lambda}$. Observe also that since the characters are class functions, $i$ pqn can be replaced by any four-tuple of distinct integers.

Proof. Consider the coefficient of $\zeta_{i}^{p} \zeta_{i}^{q}$ in $\operatorname{IM}_{\lambda}\left(\zeta, w^{\prime}, \mu\right)$. It is

$$
\sum_{\sigma} \mu_{i} \chi_{\lambda}(\sigma) w_{1}^{\sigma(1)} \cdots w_{p}^{\sigma(i)} \cdots w_{p}^{\sigma(p)} \cdots w_{i}^{\sigma(i)} \cdots w_{q}^{\sigma(q)} \cdots w_{q}^{\sigma(n)} .
$$

The monomials in that sum do not appear in $\operatorname{IM}_{\lambda}\left(w^{\prime}, \mu\right)$, so this sum must be zero. Our condition is then just that the coefficient of each monomial is equal to zero, since the monomial to which a permutation $\sigma$ contributes does not change when we compose it on the right with some element of $\langle(i p),(q n)\rangle$. 
We conclude:

Proposition 3.4.2. $P_{n}=\left\{1^{n}, 21^{n-2}\right\}$.

Proof. We know that both partitions are contained in $P_{n}$, since the first one corresponds to the determinant itself, and the second one to the tangent space to the orbit of its projectivization. Therefore, by Lemma 3.4.1, it is enough to check that the vector space $C_{n}$ of class functions $F$ on $\mathfrak{S}_{n}$, such that

$$
\sum_{\tau \in(i j)(k l)} F(\sigma \tau)=0 \quad \text { for all } \sigma \text { and all } i, j, k, l,
$$

is at most two-dimensional. We prove that $F \in C_{n}$ is completely determined by its values on permutations of cycle type $\left(1^{n}\right)$ or $\left(21^{n-2}\right)$. Recall that the value of a class function $F$ on a permutation $\sigma$ only depends on its cycle type, which is encoded by a partition $\lambda$. We will thus write $F(\lambda)$ rather than $F(\sigma)$. Apply induction on the number of fixed points in $\sigma$. Suppose that $\sigma$ has at least two nontrivial cycles. Choose $i$ and $k$ in these two cycles and let $j=\sigma(i), l=\sigma(k)$, then the three permutations $\sigma(i j)$, $\sigma(k l), \sigma(i j)(k l)$ have more fixed points than $\sigma$. If $\sigma$ has a cycle of length at least four, take $i$ in this cycle and let $j=\sigma(i), k=\sigma(j), l=\sigma(k)$, to obtain the same conclusion. Finally, if $\sigma$ is of cycle type $31^{n-3}$, say with a nontrivial cycle (123), choose $i j k l=1234$. This gives the relation $2 F\left(31^{n-3}\right)+F\left(41^{n-4}\right)+F\left(21^{n-2}\right)=$ 0 . On the other hand, when $\sigma$ has cycle type $41^{n-4}$, with nontrivial cycle (1234), let $i j k l=1324$, which yields the relation $F\left(41^{n-4}\right)+F\left(221^{n-4}\right)=0$. And if $\sigma$ has cycle type $221^{n-4}$, with nontrivial cycles (12)(34), letting $i j k l=1234$ gives the relation $F\left(221^{n-4}\right)+2 F\left(21^{n-2}\right)+F\left(1^{n}\right)=0$. These three identities altogether imply that $F\left(31^{n-3}\right)$ is determined by $F\left(21^{n-2}\right)$ and $F\left(1^{n}\right)$, and then the induction argument shows that $F$ is completely determined by these two values.

Our discussion implies

$$
\widehat{T}_{\left[\operatorname{det}_{n}\right]} \mathcal{D u a l}_{2 n-2, n, n^{2}}=\widehat{T}_{\left[\operatorname{det}_{n}\right]} \operatorname{PGL}\left(M_{n}\right) \cdot\left[\operatorname{det}_{n}\right] .
$$

Theorem 3.1.1 immediately follows.

3.5. On the boundary of the orbit of the determinant. Decompose a matrix $M$ into its symmetric and skew-symmetric parts $S$ and $A$. Define a polynomial $P_{\Lambda} \in$ $S^{n}\left(M_{n}(\mathbb{C})\right)^{*}$ by letting

$$
P_{\Lambda}(M)=\operatorname{det}_{n}(A, \ldots, A, S) .
$$

This is easily seen to be zero for $n$ even so we suppose $n$ to be odd. More explicitly, $P_{\Lambda}$ can be expressed as follows. Let $\operatorname{Pf}_{i}(A)$ denote the Pfaffian of the skew-symmetric matrix, of even size, obtained from $A$ by suppressing its $i$-th row and column. Then

$$
P_{\Lambda}(M)=\sum_{i, j} s_{i j} \operatorname{Pf}_{i}(A) \operatorname{Pf}_{j}(A) .
$$


Proposition 3.5.1. The polynomial $P_{\Lambda}$ belongs to the orbit closure of the determinant. Moreover, $\overline{\mathrm{GL}(W) \cdot P_{\Lambda}}$ is an irreducible codimension one component of the boundary of $\overline{\mathrm{GL}(W) \cdot\left[\mathrm{det}_{n}\right]}$, not contained in $\operatorname{End}(W) \cdot\left[\operatorname{det}_{n}\right]$. In particular $\overline{\mathrm{dc}}\left(P_{\Lambda, m}\right)=m<$ $\operatorname{dc}\left(P_{\Lambda, m}\right)$.

Proof. The first assertion is clear: for $t \neq 0$, one can define an invertible endomorphism $u_{t}$ of $M_{n}(\mathbb{C})$ by $u_{t}(A+S)=A+t S$, where $A$ and $S$ are the skewsymmetric and symmetric parts of a matrix $M$ in $M_{n}(\mathbb{C})$. Since the determinant of a skew-symmetric matrix of odd size vanishes,

$$
\left(u_{t} \cdot \operatorname{det}_{n}\right)(M)=\operatorname{det}_{n}(A+t S)=n t \operatorname{det}_{n}(A, \ldots, A, S)+O\left(t^{2}\right),
$$

and therefore $u_{t} \cdot\left[\operatorname{det}_{n}\right]$ converges to $\left[P_{\Lambda}\right]$ when $t$ goes to zero.

To prove the second assertion, we compute the stabilizer of $P_{\Lambda}$ inside $\operatorname{GL}\left(M_{n}(\mathbb{C})\right)$. The easiest way to make this computation uses the decomposition $\mathbb{C}^{n} \otimes \mathbb{C}^{n}=$ $\Lambda^{2} \mathbb{C}^{n} \oplus S^{2} \mathbb{C}^{n}$ of the space of matrices into skew-symmetric and symmetric ones. The action of $\mathrm{GL}_{n}(\mathbb{C})$ on $M_{n}(\mathbb{C})$ by $M \mapsto g M g^{t}$ preserves $P_{\Lambda}$ up to scale, and the Lie algebra of the stabilizer of $\left[P_{\Lambda}\right]$ is a $\mathrm{GL}_{n}(\mathbb{C})$ submodule of $\operatorname{End}\left(M_{n}(\mathbb{C})\right)$. We have the decomposition into $\mathrm{GL}_{n}(\mathbb{C})$-modules:

$$
\begin{aligned}
\operatorname{End}\left(M_{n}(\mathbb{C})\right) & =\operatorname{End}\left(\Lambda^{2} \oplus S^{2}\right) \\
& =\operatorname{End}\left(\Lambda^{2}\right) \oplus \operatorname{End}\left(S^{2}\right) \oplus \operatorname{Hom}\left(\Lambda^{2}, S^{2}\right) \oplus \operatorname{Hom}\left(S^{2}, \Lambda^{2}\right) .
\end{aligned}
$$

Moreover, $\operatorname{End}\left(\Lambda^{2}\right)=\mathfrak{g l}_{n} \oplus E A$ and $\operatorname{End}\left(S^{2}\right)=\mathfrak{g l}_{n} \oplus E S$, where $E A$ and $E S$ are distinct irreducible $\mathrm{GL}_{n}(\mathbb{C})$-modules. Similarly, $\operatorname{Hom}\left(\Lambda^{2}, S^{2}\right)=\mathfrak{s} \mathfrak{I}_{n} \oplus E A S$ and $\operatorname{Hom}\left(S^{2}, \Lambda^{2}\right)=\mathfrak{s l}_{n} \oplus E S A$, where $E A S$ and $E S A$ are irreducible, pairwise distinct and different from $E A$ and $E S$. Then one can check that the modules $E A$, $E S, E A S, E S A$ are not contained in the stabilizer, and that the contribution of the remaining terms is isomorphic with $\mathfrak{g l}_{n} \oplus \mathfrak{g l}_{n}$. In particular it has dimension $2 n^{2}$, which is one more than the dimension of the stabilizer of [det $\left.{ }_{n}\right]$. This implies $\overline{\mathrm{GL}}(W) \cdot P_{\Lambda}$ has codimension one in $\overline{\mathrm{GL}(W) \cdot\left[\operatorname{det}_{n}\right]}$. Since it is not contained in the orbit of the determinant, it must be an irreducible component of its boundary. Since the zero set is not a cone (i.e., the equation involves all the variables), $P_{\Lambda}$ cannot be in $\operatorname{End}(W) \cdot \operatorname{det}_{n}$ which consists of $\mathrm{GL}(W) \cdot \operatorname{det}_{n}$ plus cones.

The hypersurface defined by $P_{\Lambda}$ has interesting properties.

Proposition 3.5.2. The dual variety of the hypersurface $Z\left(P_{\Lambda}\right)$ is isomorphic to the Zariski closure of

$$
\mathbb{P}\left\{v^{2} \oplus v \wedge w \in S^{2} \mathbb{C}^{n} \oplus \Lambda^{2} \mathbb{C}^{n}, v, w \in \mathbb{C}^{n}\right\} \subset \mathbb{P}\left(M_{n}(\mathbb{C})\right) .
$$

As expected, $Z\left(P_{\Lambda}\right)^{*}$ is close to being a Segre product $\mathbb{P}^{n-1} \times \mathbb{P}^{n-1}$. It can be defined as the image of the projective bundle $\pi: \mathbb{P}(E) \rightarrow \mathbb{P}^{n-1}$, where $E=$ 
$\mathcal{O}(-1) \oplus Q$ is the sum of the tautological and quotient bundles on $\mathbb{P}^{n-1}$, by a sublinear system of $\mathcal{O}_{E}(1) \otimes \pi^{*} \mathcal{O}(1)$. This sub-linear system contracts the divisor $\mathbb{P}(Q) \subset \mathbb{P}(E)$ to the Grassmannian $G(2, n) \subset \mathbb{P} \Lambda^{2} \mathbb{C}^{n}$.

\section{A large irreducible component of $\mathfrak{D u a l}_{k, d, N}$}

Let $S u b_{k+2}\left(S^{d} W^{*}\right)$ be the projectivization of

$$
\left\{P \in S^{d} W^{*} \mid \operatorname{dim}\left(U^{*}\right)=k+2 \text {, and } P \in S^{d} U^{*} \text { for some } U^{*} \subset W^{*}\right\}
$$

the subspace variety of hypersurfaces of degree $d$ in $\mathbb{P} W$ that are cones over some $Z^{k} \subset \mathbb{P}^{k+1} \subset \mathbb{P} W$. The reduced, irreducible variety $\operatorname{Sub}_{k+2}\left(S^{d} W^{*}\right)$ is of dimension $k+1+(k+2)(N-(k+2))$ and its ideal is generated in degree $k+3$ (see [8], §7.2).

If $[P] \in \operatorname{Sub}_{k+2}\left(S^{d} W^{*}\right)$, then $Z(P) \subset \mathbb{P} W$ is a cone with an $(N-k-1)$ dimensional vertex $\mathbb{P}\left(U^{*}\right)^{\perp}$, and $Z(P)^{*} \subset \mathbb{P} U^{*}$. In particular $\operatorname{dim}\left(Z(P)^{*}\right) \leq k$.

Proposition 4.1.1. $\operatorname{Sub}_{k+2}\left(S^{d} \mathbb{C}^{N}\right)$ is a reduced, irreducible component of Dual $_{k, d, N}$.

Proof. Let $W^{*}=\mathbb{C}^{N}$ and let $P \in \operatorname{Sub}_{k+2}\left(S^{d} W^{*}\right)$ be a general point. Write $P \in S^{d} U^{*}$. It follows from the Kempf-Weyman desingularization described in $\S 7.2$ of $[8]$ that

$$
\widehat{T}_{[P]} \operatorname{Sub}_{k+2}\left(S^{d} W^{*}\right)=S^{d} U^{*}+\langle(u\lrcorner P) \circ \alpha\left|u \in U, \alpha \in W^{*}\right\rangle .
$$

If we choose a complement $V^{*}$ to $U^{*}$ in $W^{*}$ we may write

$$
\widehat{T}_{[P]} S_{u b} b_{k+2}\left(S^{d} W^{*}\right)=S^{d} U^{*} \oplus\langle(u\lrcorner P) \circ \alpha\left|u \in U, \alpha \in V^{*}\right\rangle .
$$

We must show

$\widehat{T}_{[P]} \operatorname{Dual}_{k, d, N} \subseteq \widehat{T}_{[P]} \operatorname{Sub}_{k+2}\left(S^{d} W^{*}\right) \subset S^{d} U^{*} \oplus S^{d-1} U^{*} \otimes V^{*} \subset S^{d}\left(U^{*} \oplus V^{*}\right)$.

Following the notation and discussion of $\S 3.3$, for $P \in \operatorname{Sub}_{k+2}\left(S^{d} W^{*}\right)$ in equation (7) we have $Q_{F}=0$, since the determinant of the Hessian on any $k+3$-plane vanishes, and we conclude that $H_{\pi, w}(X)=0$ for all $[w] \in Z(P)$ and for all $X \in V$. This says the degree $d-2$ hypersurface $Z\left(H_{\pi,-}(X)\right)$ is contained in the irreducible degree $d$ hypersurface $Z(P)$, which implies $\pi(X, X, \cdot, \ldots, \cdot)=\frac{\partial^{2} \pi}{(\partial X)^{2}}=0$ for all $X \in V$, i.e., $\widehat{T}_{[P]} \operatorname{Dual}_{k, d, N} \subseteq S^{d} U^{*} \oplus S^{d-1} U^{*} \otimes V^{*}$. To obtain the restrictions on the term in $S^{d-1} U^{*} \otimes V^{*}$ we must consider the term of order two in $\epsilon$ in the expansion of $\operatorname{det}\left(\left.H_{P_{\epsilon}}\right|_{F}\right)=P_{\epsilon} Q_{\epsilon, F}$. With our choice of splitting we may identify 
$U=\left(V^{*}\right)^{\perp} \subset W$ and take $F^{\prime}=U$. (In other words, the choice of $F^{\prime}$ is equivalent to choosing the splitting.) Note the order $\epsilon$ term also implies in this case that $Q_{F}^{\prime}=0$.

The terms on the left hand side that potentially could contribute to the $\epsilon^{2}$ coefficient are in

$$
\operatorname{det}\left(\begin{array}{cc}
\frac{\partial^{2} P}{\partial u_{i} \partial u_{j}}+\epsilon \frac{\partial^{2} \pi}{\partial u_{i} \partial u_{j}} & \epsilon \frac{\partial^{2} \pi}{\partial u_{i} \partial X} \\
\epsilon \frac{\partial^{2} \pi}{\partial u_{j} \partial X} & \epsilon \frac{\partial^{2} \pi}{(\partial X)^{2}}+\epsilon^{2} \frac{\partial^{2} \tau}{(\partial X)^{2}}
\end{array}\right) .
$$

The actual contribution is the sum of $\operatorname{det}_{k+2}\left(\left.H_{P}\right|_{U}\right) \frac{\partial^{2} \tau}{(\partial X)^{2}}$ and terms substituting two entries from $\frac{\partial^{2} \pi}{\partial u_{i} \partial X}$ for two of the columns of $\left.H_{P}\right|_{U}=\frac{\partial^{2} P}{\partial u_{i} \partial u_{j}}$. The right hand side is zero.

Choose $[w] \in Z(P) \cap Z\left(\operatorname{det}_{k+2}\left(\left.H_{P}\right|_{U}\right)\right)$, and note that we can take a basis of elements of $W$ of this form, so the first term is zero. We conclude that the column vector $\frac{\partial^{2} \pi}{\partial u_{i} \partial X}$ is a linear combination of the columns of $\frac{\partial^{2} P}{\partial u_{i} \partial u_{j}}$ which implies $\frac{\partial \pi}{\partial X}$ is a linear combination of the $\frac{\partial P}{\partial u_{i}}$, i.e. that $\left.\frac{\partial \pi}{\partial X}=u\right\lrcorner P$ for some $u \in U$ which is what we needed to prove.

\section{References}

[1] P. Buergisser, J. M. Landsberg, L. Manivel, and J. Weyman, An overview of mathematical issues arising in the geometric complexity theory approach to VP $\neq$ VNP. SIAM J. Comput. 40 (2011), no. 4, 1179-1209. Zbl 05968856 MR 2861717

[2] I. M. Gel'fand, M. M. Kapranov, and A. V. Zelevinsky, Discriminants, resultants, and multidimensional determinants. Math. Theory Appl., Birkhäuser, Boston, Mass., 1994. Zbl 0827.14036 MR 1264417

[3] T. Mignon and N. Ressayre, A quadratic bound for the determinant and permanent problem. Internat. Math. Res. Notices 2004 (2004), no. 79, 4241-4253. Zbl 1081.15003 MR 2126826

[4] D. K. Mulmuley and M. Sohoni, Geometric complexity theory. I. An approach to the P vs. NP and related problems. SIAM J. Comput. 31 (2001), no. 2, 496-526. Zbl 0992.03048 MR 1861288

[5] D. K. Mulmuley and M. Sohoni, Geometric complexity theory. II. Towards explicit obstructions for embeddings among class varieties. SIAM J. Comput. 38 (2008), no. 3, 1175-1206. Zbl 1168.03030 MR 2421083

[6] P. Pragacz, A note on the elimination theory. Nederl. Akad. Wetensch. Indag. Math. 49 (1987), no. 2, 215-221. Zbl 0415.68008 MR 0526203

[7] L. G. Valiant, The complexity of computing the permanent. Theoret. Comput. Sci. 8 (1979), no. 2, 189-201. Zbl 1075.13007 MR 1988690

[8] J. Weyman, Cohomology of vector bundles and syzygies, Cambridge Tracts in Math. 149, Cambridge University Press, Cambridge 2003. Zbl 1075.13007 MR 1988690

Received April 27, 2010 
Joseph M. Landsberg, Department of Mathematics, Texas A\&M University, Mailstop 3368, College Station, TX 77843-3368 U.S.A.

E-mail: jml@math.tamu.edu

Laurent Manivel, Institut Fourier, UMR 5582 du CNRS, Université de Grenoble I, BP 74, 38402 Saint-Martin d'Hères, France

E-mail: laurent.manivel@ujf-grenoble.fr

Nicolas Ressayre, Institut Camille Jordan (ICJ), Université Claude Bernard Lyon 1, 43 boulevard du 11 novembre 1918, 69622 Villeurbanne cedex, France

E-mail: ressayre@math.univ-lyon1.fr 